\title{
Characterization and Empirical Modelling of Sliding Wear on Sintered Aluminium-Graphite Composites
}

\author{
Amrishraj Doraisamy and Senthilvelan Thiagarajan \\ Department of Mechanical Engineering, Pondicherry Engineering College, Puducherry 605014, India \\ Correspondence should be addressed to Amrishraj Doraisamy; amriklu@gmail.com
}

Received 5 June 2013; Revised 14 December 2013; Accepted 11 February 2014; Published 30 March 2014

Academic Editor: S. Aravindan

Copyright (C) 2014 A. Doraisamy and S. Thiagarajan. This is an open access article distributed under the Creative Commons Attribution License, which permits unrestricted use, distribution, and reproduction in any medium, provided the original work is properly cited.

\begin{abstract}
Aluminium-graphite composites were synthesized using powder metallurgy route. Graphite was added as reinforcement in the range of 0,3 , and 6 weight $\%$ and composites were prepared by P/M. Microstructural analysis of the newly synthesized composites was carried out using SEM. The hardness of the composites was studied using Vickers microhardness tester, by applying a load of $1 \mathrm{~kg}$ for $5 \mathrm{sec}$. Also the amount of porosity was determined. Further the wear test was conducted on the sintered specimens using pin-on-disc wear apparatus according to ASTM-G99 standards. A regression model was developed to predict the wear rate of the specimen. Then the worn images were studied using SEM based on response surface methodology in order to understand the various wear mechanisms involved. The study revealed that mild wear, oxidational wear, plowing, cutting, and plastic deformation are the main mechanisms responsible for causing the wear.
\end{abstract}

\section{Introduction}

Recently new materials assumed the important position in engineering field. Those materials are expected to fulfill the demand of almost all engineering applications by possessing tremendous mechanical and physical properties. In present situation, various scientists and researchers are developing new engineering materials which will be compatible for versatile applications. Various materials have been combined with each other and yield intended properties in each and every part of the world; that is, the development of new materials gives another unique property which are different from their base materials. From the ancient age, this idea has been effective for mankind. Composite materials make this concept true and adding reinforcement in a matrix of this material contributes to enhanced properties. However neither with matrix or with reinforcement alone can the desired properties be fulfilled nor it is possible with a combination of these two constituents called composites. Composites are exciting materials which find increasing applications in aerospace, defence, transportation, communication, power, electronics, recreation, sporting, and numerous other commercial and consumer products. In recent years, a considerable amount of work has been carried out on the metal matrix-graphite particle composites which exhibit low friction, low wear, and excellent antiseizure properties. In these composites, graphite presumably improves tribological properties by forming a graphite-rich transfer film on the sliding surfaces [1]. Considerable efforts have been made to incorporate lubricating particles in aluminium matrices to improve wear resistance. Aluminium-graphite particulate composite exhibits its potential to act as a self-lubricating material with improved resistance to wear, machinability, and delayed onset of severe wear and seizure $[2,3]$.

Metal matrix composites (MMCs) represent new generation engineering materials. With bicontinual development in fabrication technique, more MMCs have been found to be suitable to replace some of the conventional metallic monolithic alloys such as the various grades of $\mathrm{Al}$ alloys in application, where light weight and energy saving are important design considerations $[4,5]$. Even though they have been recently used they have more tremendous effect due to their useful properties like specific strength, specific stiffness, wear resistance, corrosion resistance, elastic modulus, and so forth $[4,6]$. Metal matrix composites (MMCs) combine both metallic properties (ductility and toughness) 
and ceramic properties (high strength and modulus) possess greater strength in shear and compression and high service temperature capabilities. The extensive use of MMCs in aerospace, automotive industries, and structural applications has increased over the past two decades due to the availability of inexpensive reinforcements and cost effective processing routes which give rise to reproducible properties. The frontier zone between the matrix and reinforcement phase (interface or interphase) is an essential part of MMC. Bonding between the two phases develops from interfacial frictional stress, physical and chemical interaction, and thermal stresses due to mismatch in the coefficients of thermal expansion of the matrix and reinforcement $[1,7]$. During the design of a MMC the underlying interfacial phenomenon which governs the thermal, electrical, and mechanical properties is of utmost importance. The recent recognition that addition of ceramic reinforcements enables manipulation of physical and mechanical properties of MMCs has led to increasingly widespread use of these materials in electronic packaging and thermal-management applications. Recent market forecasts suggest the prospect for accelerating growth of MMC use as the materials are more widely understood and are cheap, suggesting a bright future for this class of materials.

Research and development on MMCs have increased considerably in the last 10 years due to their improved modulus, strength, wear resistance, thermal resistance, and fatigue resistance and improved consistency in properties and performance in general compared to the unreinforced matrix alloys [8]. The reinforcements are added extrinsically or formed internally by chemical reaction. The properties of MMCs depend on the properties of matrix material, reinforcements, and the matrix-reinforcement interface.

\section{Experimental Details}

2.1. Materials and Methodology. The composites were produced with metal powders as raw materials. Atomized pure aluminium powder was used with average particle size of $45 \mu \mathrm{m}$. The average particle size of the graphite powder was $50 \mu \mathrm{m}$.

2.1.1. Preparation of Composites. The composites were produced using powder metallurgy route, which consists of mixing, compaction, and sintering.

Mixing of Powders. The powders were mixed in a beaker and stirred for a time period of 30 minutes.

Compaction. The powders were filled into a die cavity and compaction was done using uniaxial pressing. The die consists of a die cavity, an upper punch, and a lower punch and ejector rod. First the die cavity was placed on the bottom punch and powders are now filled into the die cavity. Then the upper punch was placed on the die and pressure was applied on the upper punch in order to obtain the green compact. The compacts obtained were of $\varnothing 15 \times 25 \mathrm{~mm}$. The compaction load was varied according to the density required. The various loads given to obtain different density are shown below:

$$
\begin{aligned}
& \text { Low density }(2.47 \mathrm{~g} / \mathrm{cc})=\text { Load- } 2 \mathrm{Mpa} \\
& \text { Medium Density }(2.56 \mathrm{~g} / \mathrm{cc})=\text { Load-5 Mpa } \\
& \text { High density }(2.7 \mathrm{~g} / \mathrm{cc})=\text { Load- } 7 \mathrm{Mpa}
\end{aligned}
$$

Sintering. Sintering (heat treatment) was carried out in a muffle furnace. Prior to sintering the green compacts were given ceramic coating in order to prevent oxidation during the sintering process. The compacts were first heated to a temperature of $625^{\circ} \mathrm{C}$, held at that temperature for about $60 \mathrm{~min}$, and then allowed to cool slowly in the furnace itself.

2.2. SEM Analysis. With an objective to study the microstructure of the green composites SEM analysis was done. The SEM images were taken at Central Instrumentation Facility (CIF) in Pondicherry University.

2.3. Microhardness Test. The hardness of the newly synthesized composites was measured using Vickers hardness testing machine. Five readings were taken for each sample in order to determine the repeatability of the machine.

2.4. Porosity Measurement. Porosity has been measured using the following formula:

$$
P=1-\left(\frac{d_{s}}{d_{t}}\right),
$$

is porosity, $d_{s}$ is sintered density, and $d_{t}$ is theoretical density.

2.5. Density Measurement. Density of the composites was measured using Archimedes principle.

2.6. Pin-on-Disc Wear Test. Wear test was conducted on the specimens prepared according to ASTM G99 standards. The tests were conducted according to the runs obtained from the design matrix by varying load applied as well as the total sliding distance. The sliding velocity was kept constant as $0.3 \mathrm{~m} / \mathrm{s}$.

2.7. SEM Image of Worn Surface. In order to understand various mechanisms involved in wear, SEM analysis was done. The SEM images were taken at Central Instrumentation Facility (CIF) in Pondicherry University. EDX analysis was also taken on the worn surface to see the various elements present to understand the type of wear occurred.

\section{Design of Experiments}

Design of experiments (DOE) or experimental design is the design of any information-gathering exercises where variation is present, whether under the full control of the experimenter or not. However, in statistics, these terms are usually used for controlled experiments. Formal planned experimentation is often used in evaluating physical objects, chemical formulations, structures, components, and materials. Other types of study, and their design, are discussed in 
the articles on opinion polls and statistical surveys (which are types of observational study), natural experiments, and quasiexperiments.

3.1. Response Surface Methodology. In statistics, response surface methodology (RSM) explores the relationships between several explanatory variables and one or more response variables. The method was introduced by G. E. P. Box and K. B. Wilson in 1951. The main idea of RSM is to use a sequence of designed experiments to obtain an optimal response. Box and Wilson suggest using a second-degree polynomial model to do this. They acknowledge that this model is only an approximation but use it because such a model is easy to estimate and apply, even when little is known about the process.

Response surface methodology is a collection of mathematical and statistical techniques useful for modelling and analysis of problems in which a response of interest is influenced by several variables and the objective is to optimize this response. In most of the RSM problems the form of the relationship between the response and the independent variables is unknown. Thus the first step in RSM is to find a suitable approximation for true functional relationship between $y$ and set of independent variables. Usually a low order polynomial in some region of independent variables is employed. If the response is well modeled by a linear function of the independent variables then the approximation function is the first-order model. If there is a curvature in the system then a polynomial of higher degree must be used such as the second-order model. Almost all RSM problems use one or both of these models. The eventful objective of the RSM is to determine the optimum operating conditions for the system or to determine a region of the factor space in which operating requirements are satisfied.

3.2. Design Matrix from RSM. The design matrix was obtained by selecting four factors and three levels by using RSM using DESIGN EXPERT 8.0.7.1.

\section{Results and Discussion}

4.1. SEM Analysis. SEM image (Figure 1(a)) depicts that the microstructure consists of large volume of pores which was prepared with low density. As the graphite content gets increased the amount of pores gets reduced considerably as shown in Figure 1(b). But still it consists of pores because of the low compaction load applied during compaction. As the density is increased, the porosity gets reduced as grain gets flattened due to high load. This can be seen in Figure 1(c). Due to higher load which had been applied during the graphite particles gets agglomerated and forms a film over the base metal, which will be very much useful in reducing the wear. This is evident from Figure 1(d).

4.2. Microhardness Test. From Figure 2, it can be seen that, as the $\%$ of graphite content is increased, the hardness of the composites gets decreased. This is attributed to the soft nature
TABLE 1: Various parameters and their levels in pin-on-disc test.

\begin{tabular}{lccc}
\hline Parameter & High & Medium & Low \\
\hline Load $(\mathrm{N})$ & 50 & 30 & 10 \\
Sliding distance $(\mathrm{m})$ & 200 & 120 & 40 \\
Density $(\mathrm{g} / \mathrm{cc})$ & 2.7 & 2.56 & 2.47 \\
\% of reinforcement & 6 & 3 & 0 \\
\hline
\end{tabular}

of the graphite particles due to which it makes the indenter hit the composite easily.

It is clearly seen from Figure 3 that as the density increases the hardness of the composites increases. This is due to the fact that at higher compaction load the grain structure undergoes strain hardening effect due to which the hardness is increased.

4.3. Porosity Measurement. It has been observed from Figure 4 that as the graphite content is increased the porosity is reduced. This is attributed to the softer nature of the graphite particles which easily fills the voids present in the structure.

Volume of pores gets decreased as the density is increased. This is attributed to the high compaction load due to which the grains get flattened thereby reducing the voids. This can be observed from Figure 5.

4.4. Pin-on-Disc Wear Test. From Figure 6 it is inferred that as the load increases the wear rate increases. As the load increases, the contact pressure between the asperities increases, thereby increasing the wear. The wear rate also increases with increase in sliding distance. As sliding distance increases the area of sliding contact gets increased thereby leading to increased wear. This is seen from Archard wear model

$$
W=K * p * S
$$

From Figure 7 it can be observed that as the load increases the wear rate increases. As the load increases the contact pressure between the asperities increases thereby increasing the wear. With increase in graphite content the graphite particles get agglomerated thereby forming a thin film thereby reducing the wear. As the density increases the hardness increases and the pores get reduced thereby improving the wear resistance of the composites which can be observed from Figure 11.

Wear test was conducted on the specimens prepared according to ASTM G99 standards. The tests were conducted according to the runs obtained from the design matrix in Table 2 by varying load applied as well as the total sliding distance. The sliding velocity was kept constant as $0.3 \mathrm{~m} / \mathrm{s}$. The various parameters and their levels taken are as shown in Tables 1 and 3.

The hardness values of the composites at different graphite content and different densities are shown in Table 4.

From Figure 8 it can be observed that as the load increases the wear rate increases. As the load increases, the contact pressure between the asperities increases thereby increasing 


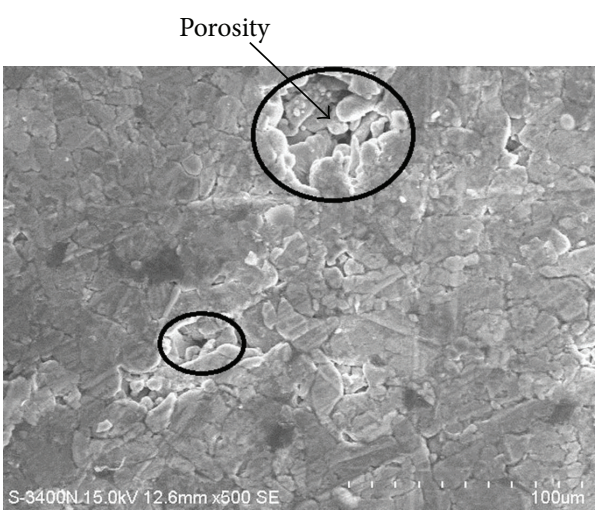

(a)

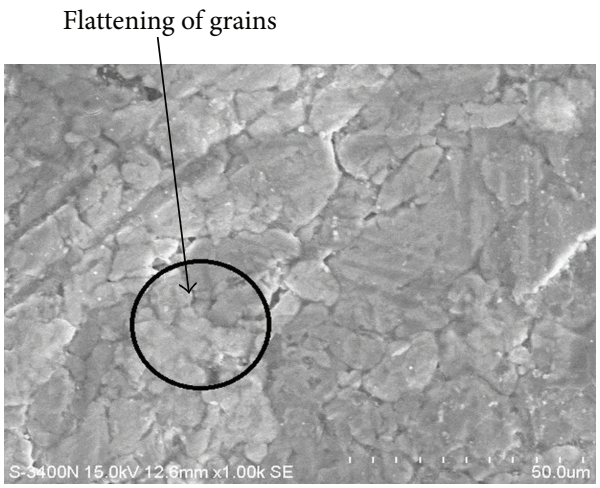

(c)

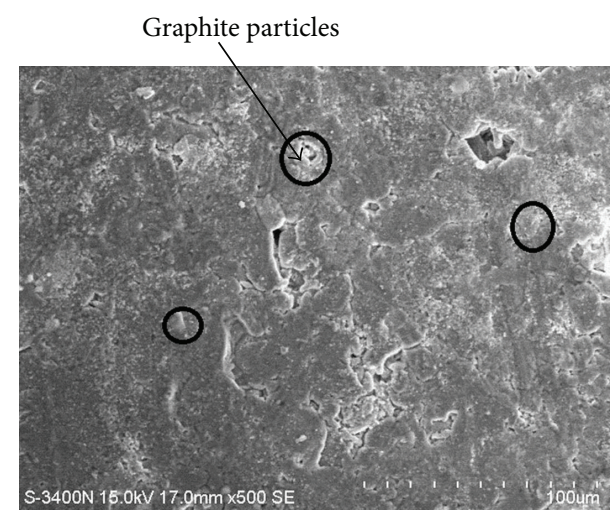

(b)

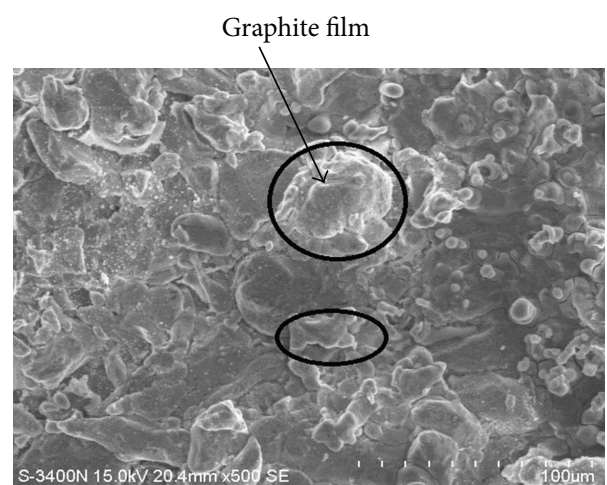

(d)

FIGURE 1: SEM images of aluminium-graphite composites: (a) $\mathrm{Al}+0 \%$ graphite-low density; (b) $\mathrm{Al}+6 \%$ graphite-low density; (c) $\mathrm{Al}+$ $0 \%$ graphite-high density; (d) $\mathrm{Al}+6 \%$ graphite-high density.

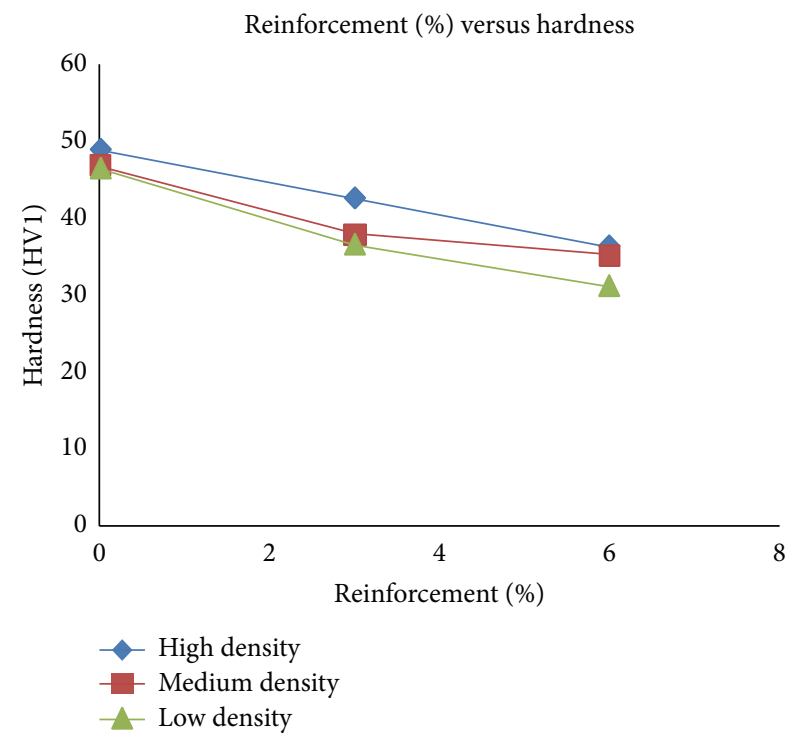

FIGURE 2: Variation of hardness of Al-Gr composites with varying graphite content.

the wear. As the density increases the hardness increases and the pores get reduced thereby reducing wear rate.

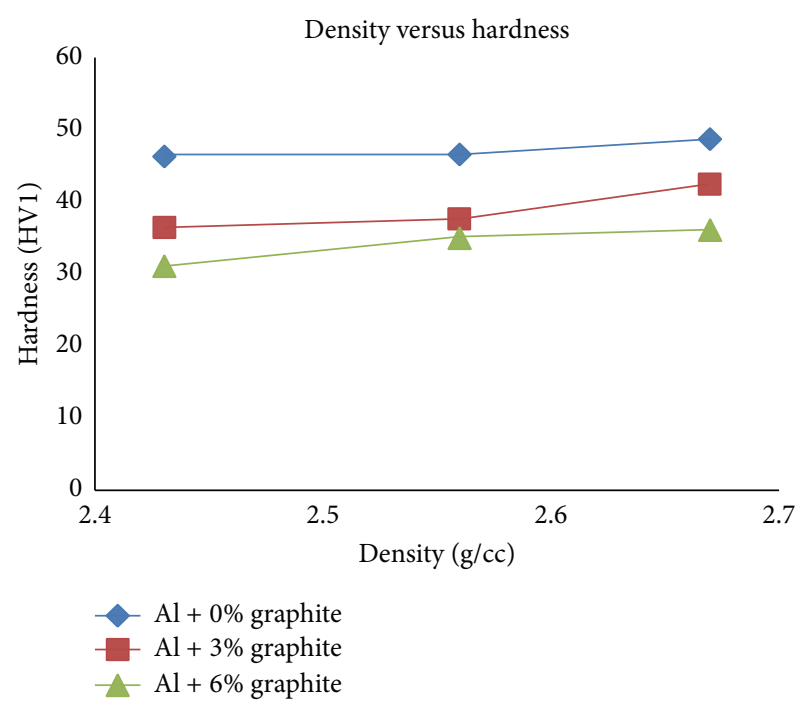

FIGURE 3: Variation of hardness of Al-Gr composites with varying density.

From Figure 9 it is concluded that the wear rate increases with increase in sliding distance. As sliding distance increases the area of sliding contact gets increased thereby leading 


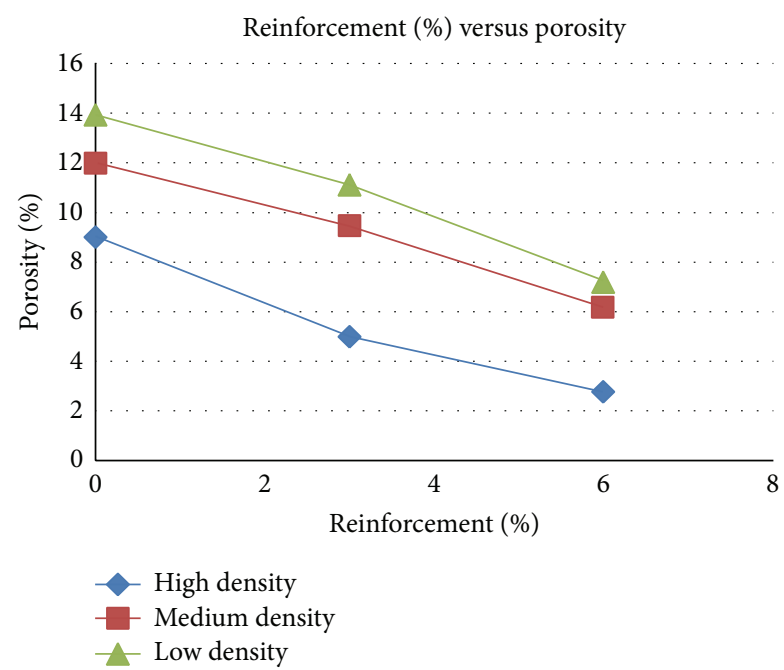

FIGURE 4: Variation of porosity of Al-Gr composites with varying graphite contents.

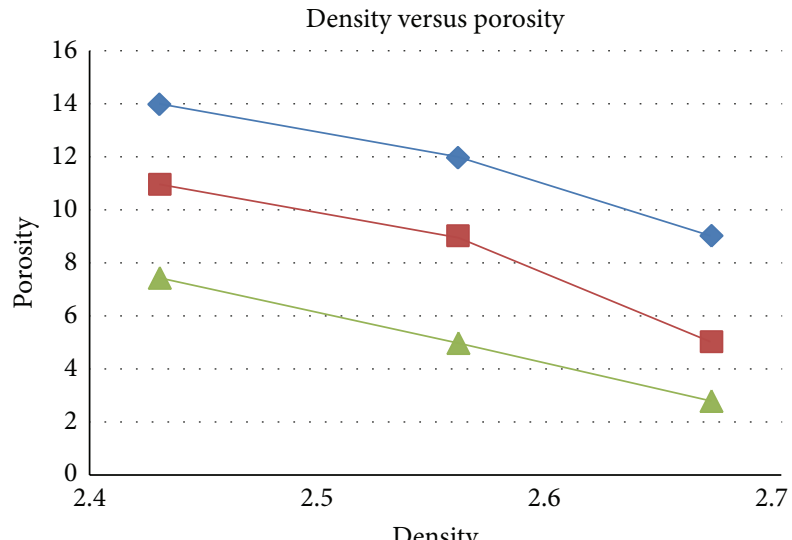

$\mathrm{Al}+0 \%$ graphite

$\mathrm{Al}+3 \%$ graphite

$\mathrm{Al}+6 \%$ graphite

FIGURE 5: Variation of porosity of Al-Gr composites with varying density.

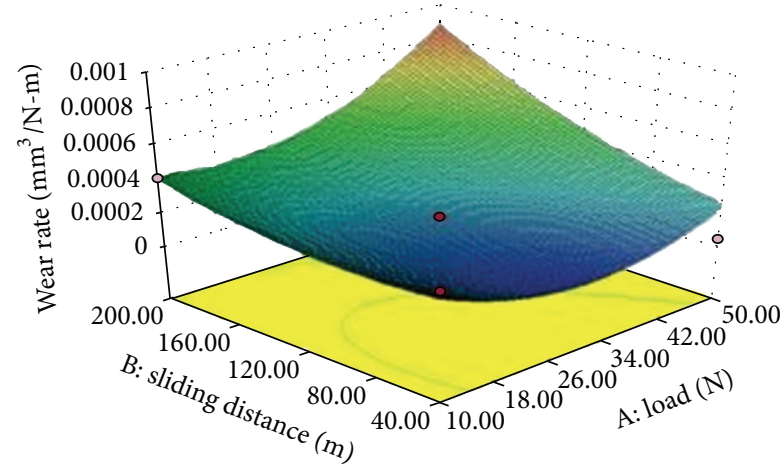

FIGURE 6: Variation of specific wear rate of Al-Gr composites with varying load and sliding distance.

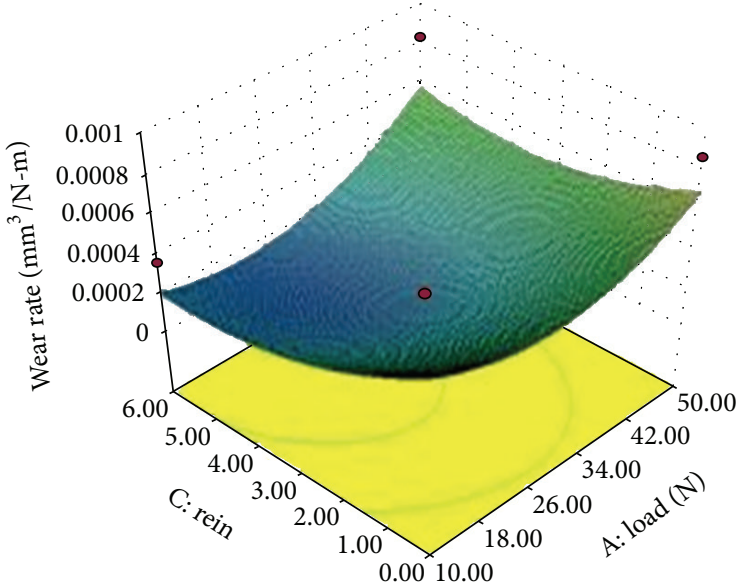

FIGURE 7: Variation of wear rate of Al-Gr composites with varying load and graphite content.

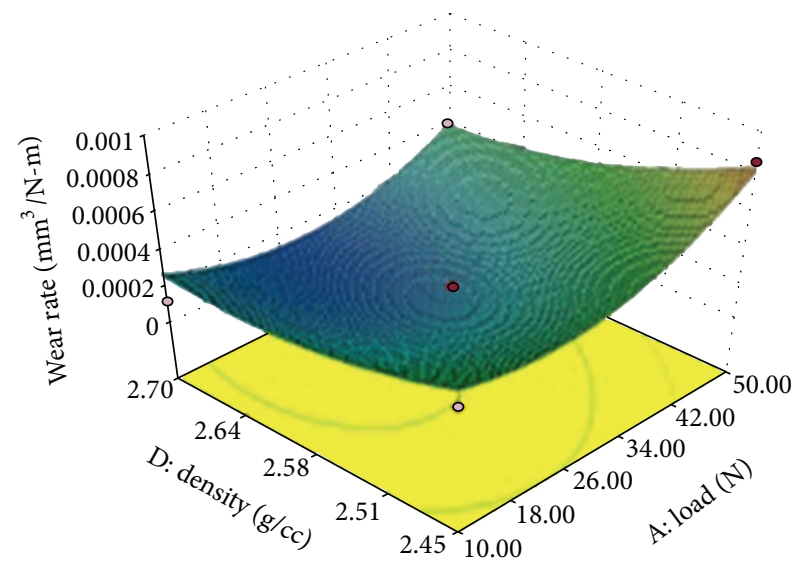

FIGURE 8: Variation wear rate of Al-Gr composites with varying load and density.

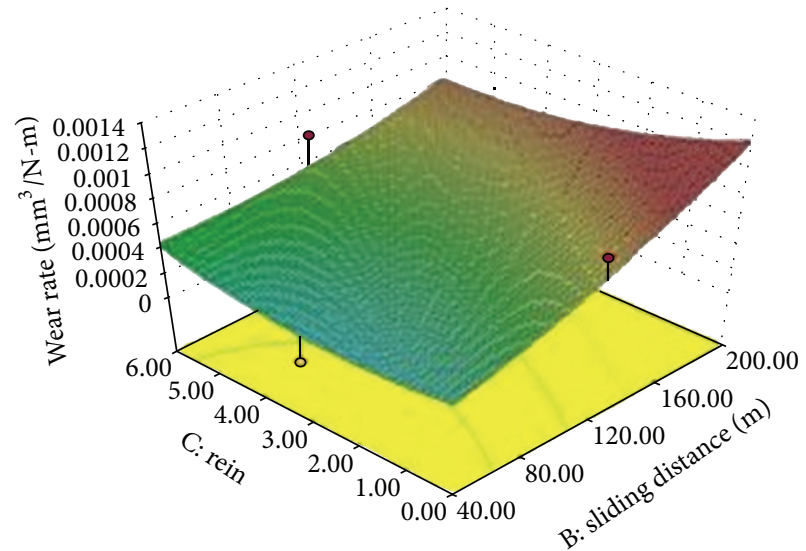

FIGURE 9: Variation of wear rate of Al-Gr composites with distance and graphite content. 


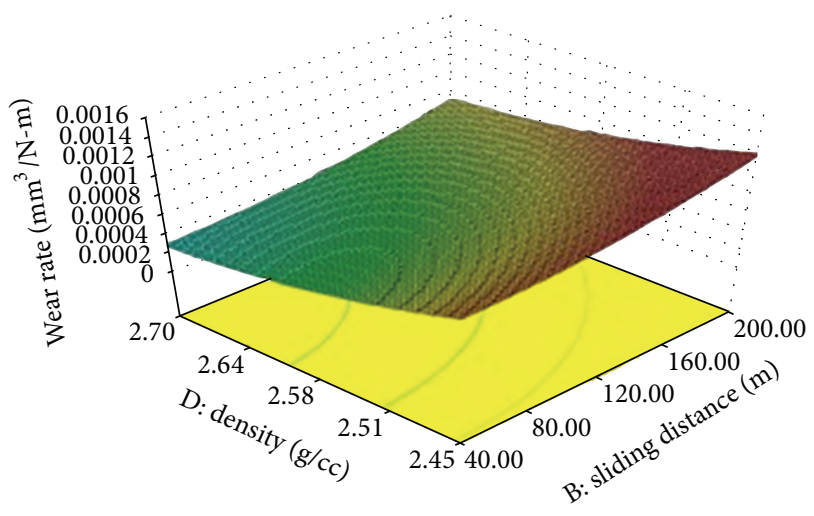

FIGURE 10: Variation of wear rate of Al-Gr composites with distance and density.

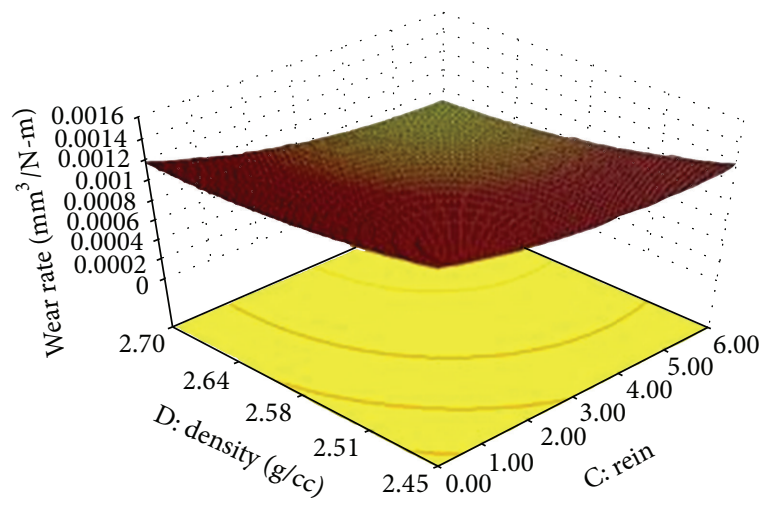

FIGURE 11: Variation of wear rate of Al-Gr composites with graphite content and density.

to increased wear. With increase in graphite content the graphite particles get agglomerated thereby forming a thin film thereby reducing the wear.

Figure 10 proves that the wear rate increases with increase in sliding distance. As sliding distance increases the area of sliding contact gets increased thereby leading to increased wear. As the density increases the hardness increases and the pores get reduced thereby improving the wear resistance of the composites.

With increase in graphite content the graphite particles get agglomerated thereby forming a thin film thereby reducing the wear. As the density increases the hardness increases and the pores get reduced thereby improving the wear resistance of the composites.

4.5. Regression Model. Regression equations obtained from RSM analysis based on experiments for the wear loss are expressed in

$$
\begin{aligned}
\text { Wear rate }= & 1.812 e-4+1.18 e-4 * \text { Load }+1.984 e \\
& -4 * \text { distance }-1.292 e-4 * \text { rein } \\
& -1.588 e-4 * \text { density }+2.014 e-4 *\left(\operatorname{load}^{2}\right) .
\end{aligned}
$$

\begin{tabular}{|c|c|c|c|}
\hline Load $(\mathrm{N})$ & $\begin{array}{c}\text { Sliding } \\
\text { distance }(\mathrm{m})\end{array}$ & $\begin{array}{c}\% \text { of } \\
\text { reinforcement }\end{array}$ & Density (g/cc) \\
\hline $\begin{array}{ll}-1 \\
\end{array}$ & 0 & -1 & 0 \\
\hline-1 & 0 & 0 & 1 \\
\hline 0 & 0 & 1 & 1 \\
\hline 0 & 1 & 0 & -1 \\
\hline 0 & -1 & 0 & 1 \\
\hline 0 & 0 & 0 & 0 \\
\hline 0 & 1 & 1 & 0 \\
\hline 1 & 0 & 0 & 1 \\
\hline 1 & 0 & 1 & 0 \\
\hline 1 & 1 & 0 & 0 \\
\hline 0 & 1 & -1 & 0 \\
\hline 0 & -1 & 0 & -1 \\
\hline 0 & 0 & 0 & 0 \\
\hline-1 & 0 & 1 & 0 \\
\hline 0 & 0 & 0 & 0 \\
\hline 0 & 1 & 0 & 1 \\
\hline-1 & -1 & 0 & 0 \\
\hline 1 & 0 & 0 & -1 \\
\hline 1 & 0 & -1 & 0 \\
\hline 0 & -1 & -1 & 0 \\
\hline 0 & -1 & 1 & 0 \\
\hline 0 & 0 & 1 & -1 \\
\hline-1 & 0 & 0 & -1 \\
\hline 1 & -1 & 0 & 0 \\
\hline 0 & 0 & -1 & -1 \\
\hline 0 & 0 & 0 & 0 \\
\hline 0 & 0 & 0 & 0 \\
\hline-1 & 1 & 0 & 0 \\
\hline 0 & 0 & -1 & 1 \\
\hline
\end{tabular}

TABLE 2: Design matrix showing all experimental runs.

TABle 3: Parameters and their control levels.

\begin{tabular}{lcccc}
\hline \multirow{2}{*}{ Parameters } & \multicolumn{2}{c}{ Range } & \multicolumn{2}{c}{ Codes } \\
& High & Low & High & Low \\
\hline Load (N) & 50 & 10 & 1 & -1 \\
Sliding distance $(\mathrm{m})$ & 200 & 40 & 1 & -1 \\
\% of reinforcement & 6 & 0 & 1 & -1 \\
Density (g/cc) & 2.7 & 2.45 & 1 & -1 \\
\hline
\end{tabular}

Regression analysis indicates that the second-order regression model adequately represents wear rate in terms of process variables. Since the coefficients of the applied load and the sliding distance are positive, the weight loss increases with increasing the applied load and increasing the sliding distance.

4.6. SEM Image of Worn Surface. From the microstructural image shown in Figure 12(a) it can be said that at low loading condition mild abrasive wear, plowing, and oxidational 


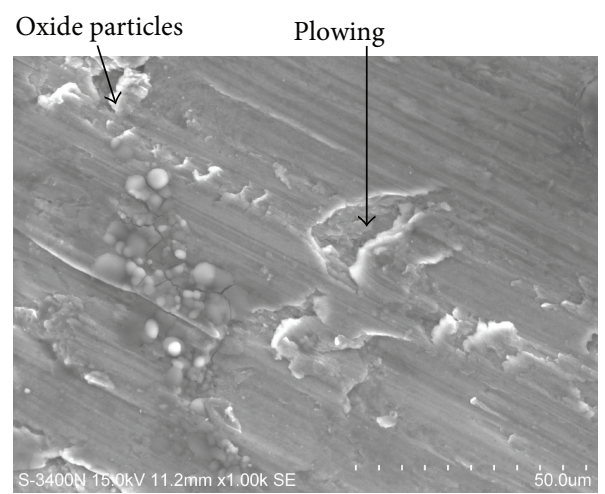

(a)

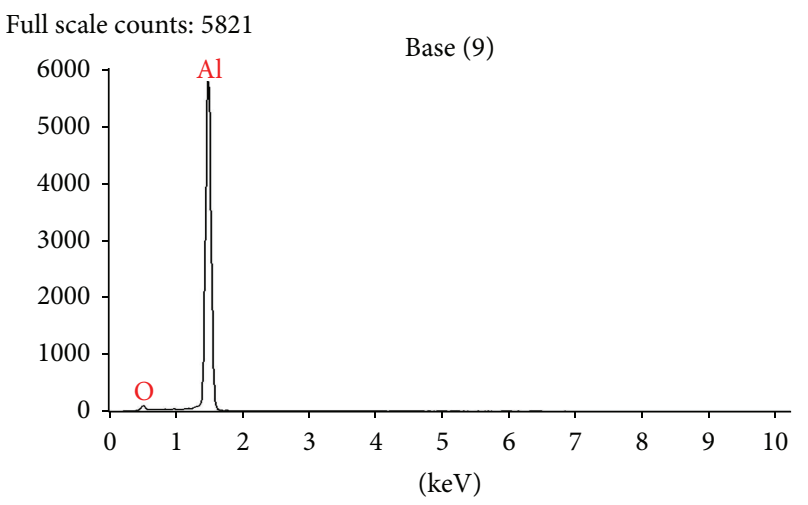

(b)

FIGURE 12: (a) SEM and (b) EDX analysis of worn surface of $\mathrm{Al}+0 \%$ graphite; load-10 N.

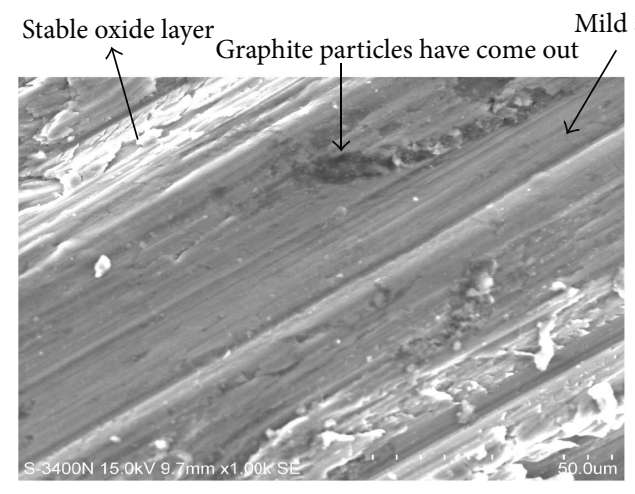

(a)

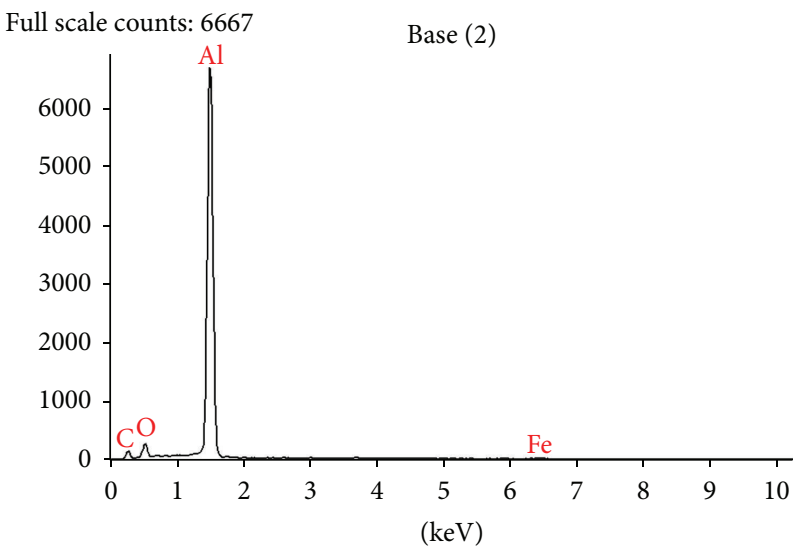

(b)

Figure 13: (a) SEM and (b) EDX analysis of worn surface of $\mathrm{Al}+6 \%$ graphite; load-10 N.

TABLE 4: Microhardness of the composites.

\begin{tabular}{lcc}
\hline S. number & Composite & Hardness (HV1) \\
\hline 1 & $\mathrm{Al}+$ 0\% graphite (high density) & 48.76 \\
2 & $\mathrm{Al}+$ 3\% graphite (high density) & 42.46 \\
3 & $\mathrm{Al}+6 \%$ graphite (high density) & 36.24 \\
4 & $\mathrm{Al}+$ 0\% graphite (medium density) & 46.6 \\
5 & $\mathrm{Al}+3 \%$ graphite (medium density) & 37.8 \\
6 & $\mathrm{Al}+6 \%$ graphite (medium density) & 35.2 \\
7 & $\mathrm{Al}+0 \%$ graphite (low density) & 46.4 \\
8 & $\mathrm{Al}+3 \%$ graphite (low density) & 36.5 \\
9 & $\mathrm{Al}+6 \%$ graphite (low density) & 31.1 \\
\hline
\end{tabular}

wear are the main mechanisms involved. EDX confirms the presence of oxide particles which reflects the presence of oxidational wear.

From the microstructural image shown in Figure 13 it can be said that at low loading condition mild abrasive wear, plowing, and oxidational wear are the main mechanisms

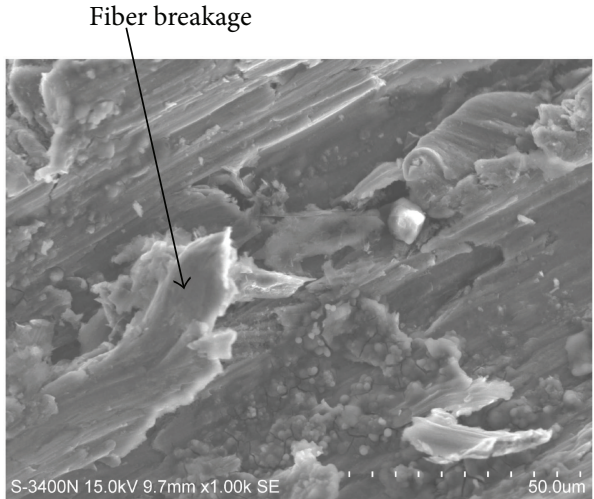

FIGURE 14: SEM analysis of worn surface of $\mathrm{Al}+0 \%$ graphite; load$50 \mathrm{~N}$.

involved. EDX confirms the presence of oxide particles which reflects the presence of oxidational wear and also graphite particles have come out due to cutting action. 


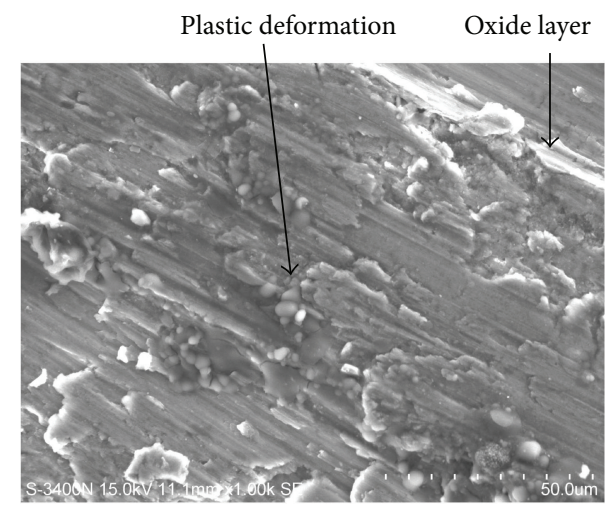

FIGURE 15: SEM analysis of worn surface of $\mathrm{Al}+6 \%$ graphite; load$50 \mathrm{~N}$.

Figure 14 reveals that at higher loads the mild wear gets transformed to severe wear thereby causing plastic deformation ultimately leading to breakage of fibers.

Figure 15 reveals that the presence of graphite particles stops the fiber breakage after the onset of plastic deformation.

\section{Conclusions}

(1) The composites were synthesized using powder metallurgy technique.

(2) SEM images reveal that at low density the porosity present is usually high. At higher density due to higher compaction load the pores get reduced considerably.

(3) From SEM image it is clear that as the graphite content is increased the porosity gets reduced. This is because the soft graphite particles occupy the voids present.

(4) Hardness test reveals that as the graphite particles get increased the hardness gets decreased. This is attributed to the soft nature of the graphite particles which makes the intender hit the composite surface easily.

(5) Hardness of the composites increases with increase in density. This is due to the strain hardening effect due to which the hardness increases.

(6) Wear test indicates that wear rate increases with increasing load and sliding distance and decreases with increase in graphite content and density.

(7) SEM morphology of worn surface shows that mild wear, severe wear due to plastic deformation, plowing, cutting, and oxidational wear are the main mechanisms involved.

(8) An empirical second-order regression model has been developed which will be useful in predicting the wear rate of composites.

\section{Conflict of Interests}

The authors declare that there is no conflict of interests regarding the publication of this paper.

\section{References}

[1] M.-J. Zhang, Y.-B. Liu, X.-H. Yang, J. An, and K.-S. Luo, "Effect of graphite particle size on wear property of graphite and $\mathrm{Al}_{2} \mathrm{O}_{3}$ reinforced AZ91D-0.8\%Ce composites," Transactions of Nonferrous Metals Society of China, vol. 18, no. 1, pp. s273-s277, 2008.

[2] Y. B. Liu, P. K. Rohatgi, and S. Ray, "Tribological charecteristics of aluminium-50\% graphite composites," Metallurgical Transactions A, vol. 24, no. 1, pp. 151-159, 1993.

[3] G. Ranganath, S. C. Sharma, and M. Krishna, "Dry sliding wear of garnet reinforced zinc/aluminium metal matrix composites," Wear, vol. 251, no. 2, pp. 1408-1413, 2001.

[4] G. B. Veeresh Kumar, C. S. P. Rao, and N. Selvaraj, "Mechanical and tribological behavior of particulate reinforced aluminum metal matrix composites-a review," Journal of Minerals \& Materials Characterization \& Engineering, vol. 10, no. 1, pp. 5991, 2011.

[5] S. Basavarajappa and G. Chandramohan, "Wear studies on metal matrix composites: a taguchi approach," Journal of Materials Science and Technology, vol. 21, no. 6, pp. 845-850, 2005.

[6] Y.-Q. Wang and J.-I. Song, "Dry sliding wear behavior of $\mathrm{Al}_{2} \mathrm{O}_{3}$ fiber and $\mathrm{SiC}$ particle reinforced aluminium based MMCs fabricated by squeeze casting method," Transactions of Nonferrous Metals Society of China, vol. 21, no. 7, pp. 1441-1448, 2011.

[7] D. Huang, W.-P. Chen, S.-Y. Zhang, and Z.-X. He, "Dry friction and wear performance of $\mathrm{SiC} 3 \mathrm{D}$ continuous ceramic frame reinforced 7075Al alloy," Transactions of Nonferrous Metals Society of China, vol. 20, no. 1, pp. 54-58, 2010.

[8] C. Y. H. Lim, S. C. Lim, and M. Gupta, "Wear behaviour of SiCpreinforced magnesium matrix composites," Wear, vol. 255, no. 1-6, pp. 629-637, 2003. 

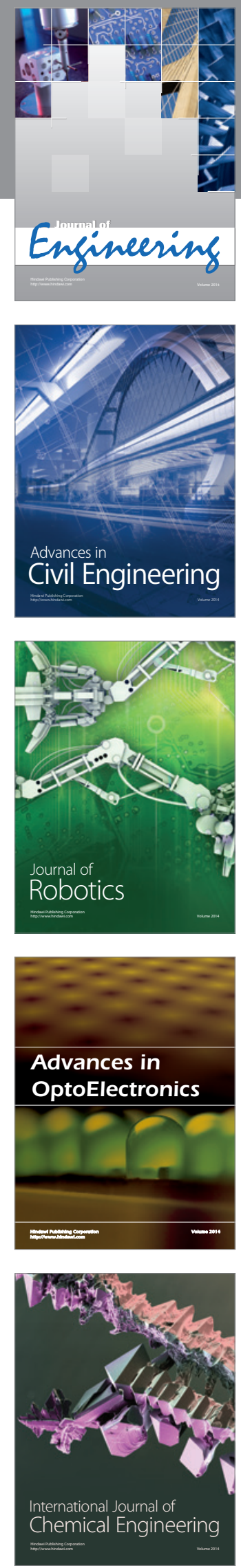

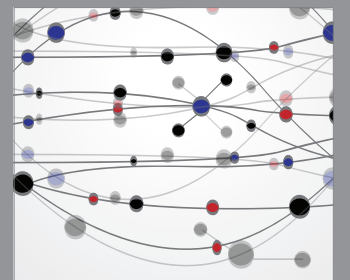

The Scientific World Journal
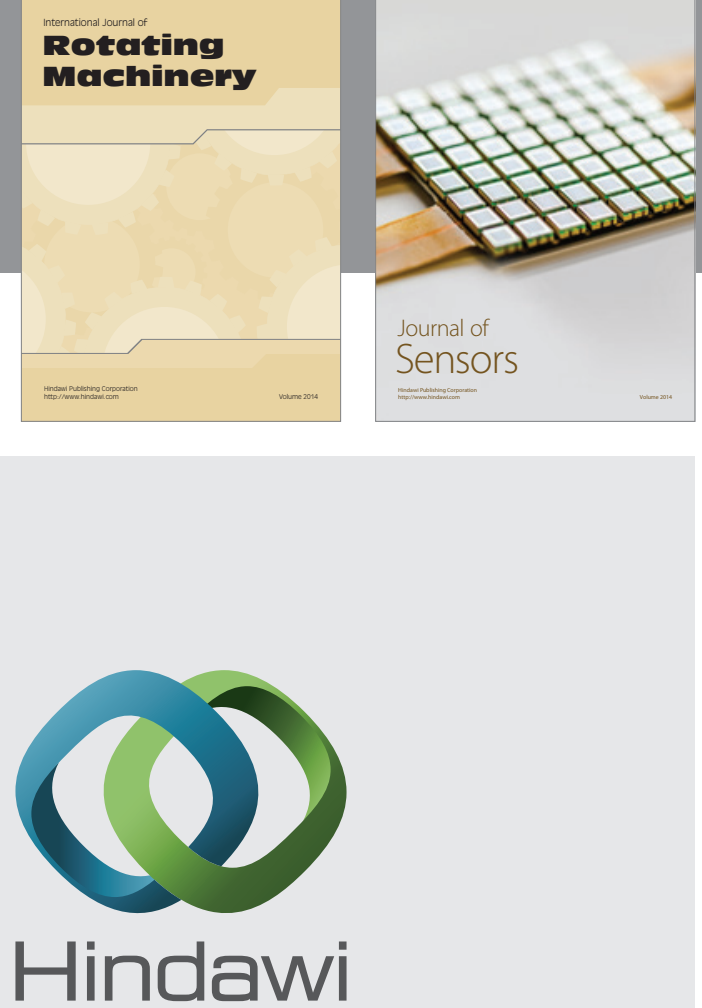

Submit your manuscripts at http://www.hindawi.com
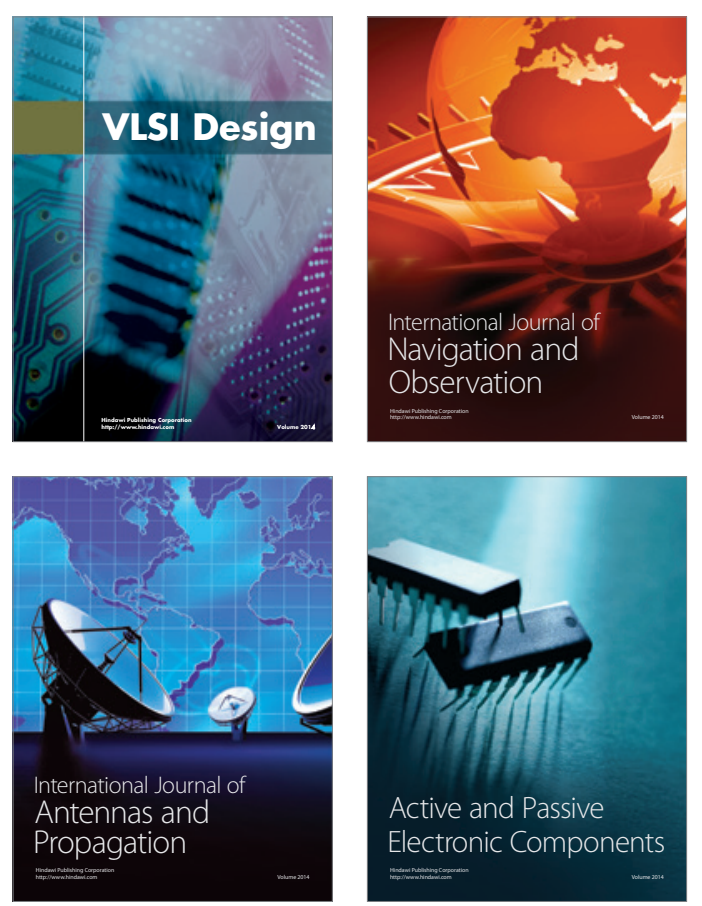
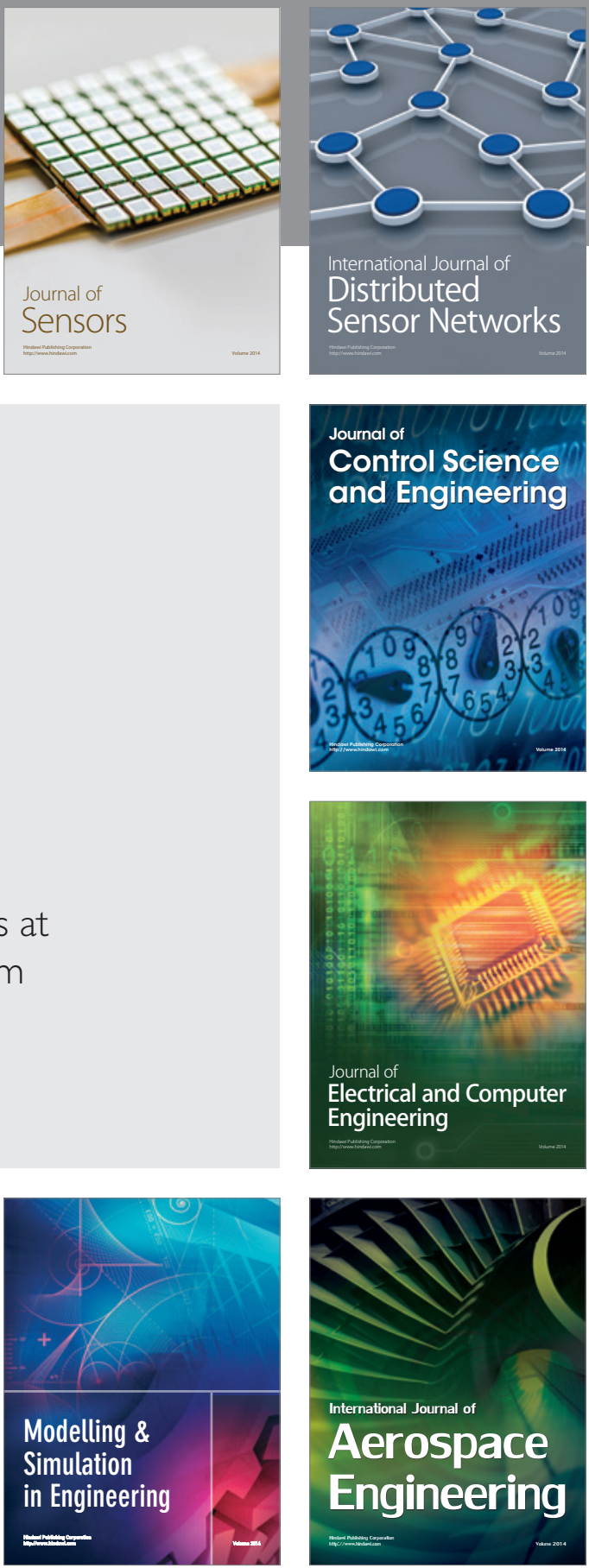

Journal of

Control Science

and Engineering
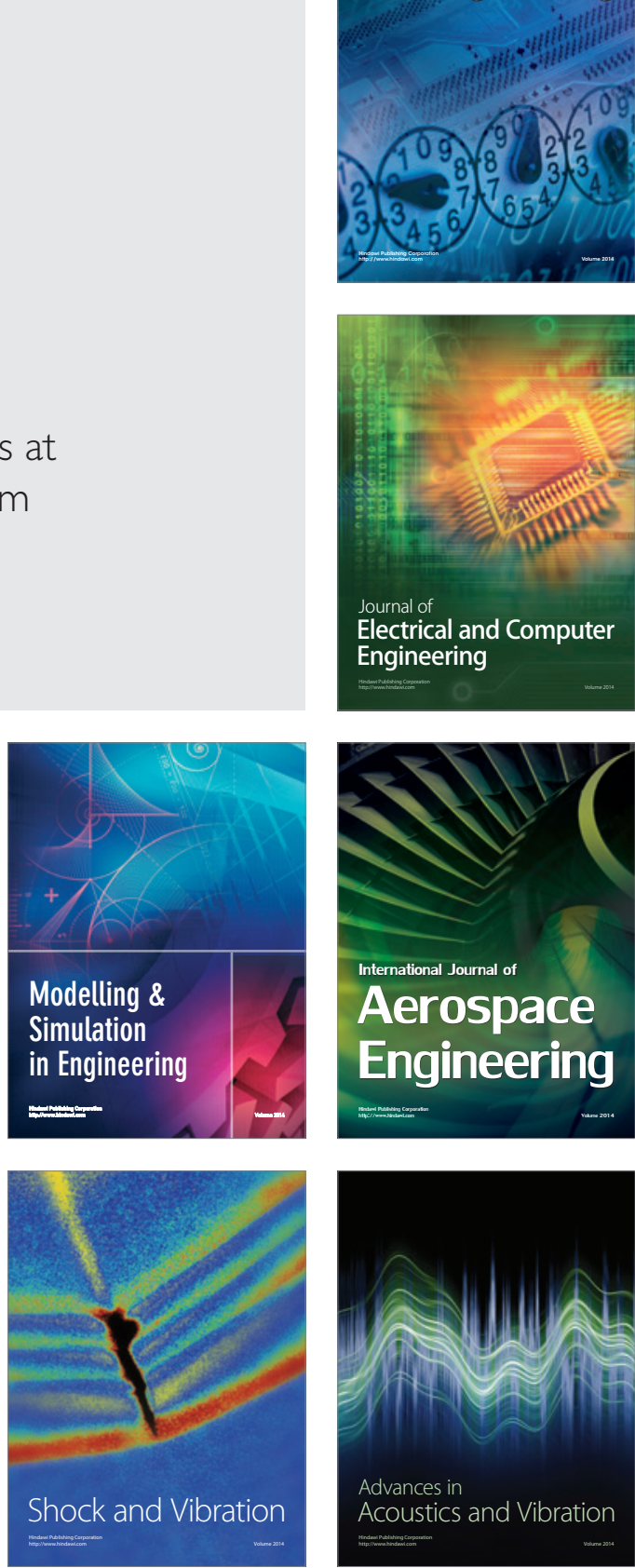